\title{
User Aware Technology: From Inter-human Awareness to Technology-User Awareness ${ }^{*}$
}

\author{
Ditte Hvas Mortensen \\ Bang \& Olufsen A/S \\ Peter Bangsvej 15, Struer, Denmark \\ Department of Psychology \\ Aarhus University, Aarhus, Denmark \\ dittehm@psy.au.dk
}

\begin{abstract}
The present project explores how the development of user-aware technology may be supported by applying knowledge from research in psychology on nonverbal aspects of human activity in context.
\end{abstract}

Research Area: Human-Technology Interaction, User-Aware Technology, Multimodal Interaction.

\section{User-Awareness}

As programmed technology is embedded into a growing array of objects demanding the user's attention, the importance of creating seamless and intuitive interaction that puts the least possible strain on the user's attention span becomes apparent. To achieve this there has been an increased interest in creating user-aware technology in recent years, especially within the field of pervasive computing. User-awareness can be understood as part of the broader concept of context-aware computing [1]. By being aware of different aspects of the user and her behavior, user-aware technology tries to adapt to the user without receiving explicit commands. A common approach for achieving this has been based on trying to predict the user's activity and wishes, by collecting extensive behavioral information about the user in question [2]. This is often a substantial task, as relevant information about a specific user is a constantly changing and in principle infinite field. Other common attempts at this are more technology driven and define user awareness more narrowly, based on what is relevant to a specific product [e.g. 3].

The present project seeks to contribute to former work by being situated between these two approaches. It focuses on examining more general principles for how technology can be aware of users, by exploring what the most basic objective indicators about the user's intentions may be, and how technology can be made aware of these. This is done through focusing on the user's nonverbal behavior, such as what the user's gaze-direction or movements indicate about her intentions.

The theoretical foundation of the project is based on research in cognitive and developmental psychology that study basic patterns of multimodal nonverbal human

* Advisors: Klaus B. Bærentsen, Søren Bech and Marianne Graves Petersen. 
behavior. At the most basic level the aspects are e.g. body movements, facial expression, gaze direction, gesture, and prosody such as tone, tempo, and pauses in speech [4-7]. This research suggests that we have large cognitive resources for expressing ourselves and perceiving the intentions of others nonverbally. This behavior is often outside of our conscious field of attention, and takes place as a constant adjustment to the activity we are involved in, to other persons and to objects around us. If a user is trying a new technology for the first time, for instance, her movements during the interaction will be slow and hesitative, but when she becomes more accustomed to the interaction they will change to being faster and goal oriented. This change in movements takes place outside of the user's conscious focus, and can often be easily interpreted by others watching her. It could be said that we act more or less instinctively on this level, rather than using a substantial amount of knowledge and model building $[8,9]$.

Because most nonverbal behavior take place outside of our conscious attention, it is important that the user-aware technology works outside of the user's conscious focus as well. The user should be able to focus on what she wishes to achieve from the technology, rather than on the operational details of the interaction. One way to help accomplishing this is by using the information given by the user's nonverbal behavior, to interpret what her intentions are, in order to narrow down the offered interaction possibilities.

\subsection{Hypothesis}

The hypothesis of the project is that technology which supports the user's interaction by being aware of, and reacting to, objective indicators of the user's intentions in nonverbal behavior is easier and more pleasurable to use.

\section{Method}

The project is divided into three parts:

1. An analysis of basic nonverbal human behavior, through qualitative pilot studies and a thorough review of psychological research in the area, to find the most relevant user information for technology

2. An analysis of the technological possibilities and limitations in creating useraware technology.

3. An experimental testing of the hypothesis by implementing the results from part 1 and 2 in concrete functions.

\subsection{Progress So Far}

In part 1 and 2 of the project, relevant technologies and aspects of nonverbal behavior are being analyzed and pilot tested individually. Nonverbal behavior can be understood as a multimodal activity. Thus, technology that reacts to the objective indicators of the user's intentions, through perception of nonverbal behavior, should function multi-modally [10]. It is however necessary to begin by analyzing the aspects of 
nonverbal behavior individually, through a review on the research done on each to identify which aspects are most basic to expressing intentions, what their precise function are as well as the technologies available to monitor them.

Through the review of the literature done so far, it has been decided to focus the further studies on two aspects of nonverbal behavior, namely gaze-direction and movements/ locomotion. These aspects are generally acknowledged as some of the most fundamental ways of expressing intentions, studies have shown that intentions expressed through gaze direction and movement can be shown and perceived very early in human development, before we are able to talk. Gaze-direction and movements, can also be implemented in a wide variety of technologies, most interfaces already require visual and haptic interaction, whereas for instance prosody would require verbal interaction through speech recognition, which is more rare $[4,11]$.

Besides the individual aspects, it has also been studied how it is possible to set up some general rules for an awareness of the user's intentions, when it is recognized that activity cannot be separated from the context that it is a part of [12]. As a part of this a qualitative pilot study of the activity of solving a task, focusing on gaze related behavior, has been carried out. This explores if an analysis of the specific action being performed can be generalized to other settings where similar actions are being performed.

\subsection{Planned Studies}

In part 3 of the project, the hypothesis will be tested experimentally. Two $\mathrm{A} / \mathrm{B}$ experiments will compare a technology that uses gaze direction and movements to perceive and react to the user's intentions, with an otherwise similar technology that does not use this. In each experiment it is planned to compare the two versions of the technology on the dependent variables precision in interaction, time, and user preference. If the assumption that it is easier to use technology that reacts to nonverbal aspects of human behavior is correct, it is to be expected that study participants are faster and more accurate with the user-aware technology, and that they prefer this form of interaction.

To ensure the validity of the experiments and in order to avoid incorrectly rejecting the hypothesis, it is very important that the stimuli are designed correctly, so that they actually represent relevant nonverbal behavior connected to the expression of intentions. This will be ensured by combining qualitative studies with an extensive review of research, and by focusing on the most basic principles of communication.

\section{Expected Contributions}

Expanding the scope of human technology interaction from explicit commands through pressing buttons, touch screens and the like, to a wider range of human behavior potentially has benefits for all types of applications. Technology that is able to perceive and react to the basic aspects of nonverbal user behavior would make use of the cognitive resources we have for perceiving and expressing these, without much increase in the cognitive load involved in the interaction, because they exist outside of the user's conscious field of attention. 


\section{References}

1. Schmidt, A., Beigl, M., Gellersen, H.-W.: There is more to context than location. Computers and Graphic 23(6), 893-901 (1999)

2. Dourish, P.: What we talk about when we talk about context. Personal and Ubiquitous Computing 8(1), 19-30 (2004)

3. Qvarfordt, P., Zhai, S.: Conversing with the User Based on Eye-Gaze Patterns. In: CHI 2005, pp. 221-230. ACM Press, Oregon (2005)

4. Turati, C., Simion, F., Milani, I., Umilta, C.: Newborns' preference for faces: What is crucial? Developmental Psychology 38(6), 875-882 (2002)

5. Brooks, R., Meltzoff, A.N.: The Importance of Eyes. Developmental Psychology 38(6), 958-966 (2002)

6. Krauss, R.M., Morrel-Samuels, P., Colasante, C.: Do conversational hand gestures communicate? Journal of Personality and Social Psychology 61(5), 743-754 (1991)

7. Henderson, J.M.: Human gaze control during real-world scene perception. Trends in Cognitive Sciences 7(11), 498-504 (2003)

8. Suchman, L.: Human-Machine Reconfigurations: Plans and Situated Actions. Cambridge University Press, New York (2006)

9. Bateson, G.: Redundancy and Coding. In: Steps to an ecology of mind: Collected essays in anthropology, psychiatry, evolution and epistemology, pp. 417-431. Jason Aronson, Northvale (1972)

10. Gibson, J.J.: The Senses Considered as Perceptual Systems. Houghton Mifflin, USA (1966)

11. Tapia, E.M., Intille, S.S., Larson, K.: Activity Recognition in the Home Using Simple and Ubiquitous Sensors. In: Ferscha, A., Mattern, F. (eds.) PERVASIVE 2004. LNCS, vol. 3001, pp. 158-175. Springer, Heidelberg (2004)

12. Barker, R.G.: Ecological Psychology. In: Concepts and Methods for Studying the Environment of Human Behavior. Stanford University Press, USA (1968) 